\section{Cognitive impairment behind the wheel}

In Jennie Russell's news article No Driving for Miss Daisy Dr. Malcolm ManSon-Hing discusses the problem of assessing driver capacity. ${ }^{1} \mathrm{He}$ states that there is no scientific standard for making the call and that some doctors are too strict and others too lenient. "That kind of variation in practice is rather unfair. ... It puts a huge strain on the physician-patient relationship," said Hing. Cognitive impairment is one of the conditions where variability and guesswork may occur. This places physicians in a difficult position if they are to rely on clinical judgments about fitness to drive. A better approach is to evaluate the effects of comorbidities, polypharmacy, age and other factors using a relevant, functional assessment of driving competence.

The British Columbia Office of the Superintendent of Motor Vehicles' (OSMV) new guide, 2010 BC Guide in Determining Fitness to Drive states that if a physician makes a diagnosis of dementia or has a suspicion that there is cognitive impairment, he may perform a SIMARD-MD test and, based on the result, refer patients to the OSMV for testing. ${ }^{2}$ The OSMV then decides whether to cancel the driver's licence.

Dr. Hing's research, or the research of others, may lead to the development of more effective tools for assessing driving capacity. For now, I know that I am responsible for making the best possible decisions for my patients. The approach outlined in the 2010 BC OSMV guidelines helps me to do that.

\section{Dorothy (Sam) Williams}

Geriatrician, Vancouver Island Health Authority, Victoria, BC

\section{References}

1. Russell J. No driving for Miss Daisy. CMAJ 2011;183:784-5.

2. 2010 BC guide in determining fitness to drive. Victoria $(\mathrm{BC})$ : Ministry of Public Safety and Solicitor General Office of the Superintendent of Motor Vehicles; 2010.

CMAJ 2011. DOI:10.1503/cmaj.111-2041

\section{Tuberculosis in Nunavut: a socioeconomic tragedy}

The editorial in the Apr. 19, 2011 issue of $C M A J^{1}$ is timely, apt and disturbing because tuberculosis is seen only in the most socioeconomically deprived communities.

The treatment of those infected is resource intensive because antibiotic treatment (six months or more) must be accompanied by robust nutritional support of the patient and monitoring of response.

The eradication of tuberculosis can only be achieved by the socioeconomic upliftment of that community.

Canada has the resources, and one hopes the will, to realize that now is the time to focus on this tragedy.

\section{Paul Anderson}

Perth, Ont.

\section{Reference}

1. Macdonald N, Hébert PC, Stanbrook MB. Tuberculosis in Nunavut: a century of failure. CMAJ 2011;183:741.

CMAJ 2011. DOI:10.1503/cmaj.111-2051

\section{Angiotensin-receptor- blocker research blocked by too many assumptions}

I question a number of assumptions made by Guertin and colleagues in their analysis of the economic impact of restricting access to angiotensin-receptor blockers (ARBs). ${ }^{1}$ Notably, that angiotensin-converting-enzyme (ACE) inhibitors are all equally effective and that all cases of patients switching from an ACE inhibitor to an ARB are due to dry cough. The authors also assume that patients were not first given ACE inhibitors on a trial basis - a very common practice. The authors' argument for economic savings presumes that problems are quickly identified - at the very next visit with the same doctor - when in reality several visits and investigations may be required to determine the source of the cough.
The stopping of medication because of side effects carries risks, and expense, not fully appreciated here. The savings estimated do not, I believe, adequately reflect that retitration of a new agent can require several visits and dose adjustments. In general ARBs are available in fewer doses for each molecule.

I think the conclusions made by Guertin and coauthors are optimistic and the amount of money saved is unclear, and possibly much less than estimated. Perhaps then, we should not conclude that the suggested strategy is always appropriate. Once ARB medication becomes generic, the opposite strategy might be more cost-effective.

\section{Daniel Lalla MD}

Cowansville Medical Clinic, Cowansville, Que.

\section{Reference}

1. Guertin JR, Jackevicius CA, Cox JL, et al. The potential economic impact of restricted access to angiotensin-receptor blockers. CMAJ 2011;183: E180-6

CMAJ 2011. DOI:10.1503/cmaj.111-2042

\section{Fighting colorectal cancer with information technology}

Colorectal cancer (CRC) screening has advanced to the forefront of contemporary preventive health care in Canada. ${ }^{1,2}$ Colonoscopy is central to CRC screening as either the initial exam or the follow-up to positive exams by other screening methods. Effective transmission of colonoscopy information is required to implement and evaluate the uptake and impact of CRC screening guidelines. ${ }^{3}$ Despite provincial CRC screening programs, mechanisms for scheduling appointments, tracking and monitoring quality indicators, reporting results and contacting patients for rescreening are either not in place or not efficient. With the global effort to conquer cancer underway, it is imperative to construct a new framework for computerized colonoscopy data collection and dissemination that meets these challenges. 